\title{
OPEN Optically transparent and very thin structure against electromagnetic pulse (EMP) using metal mesh and saltwater for shielding windows
}

\author{
Duy Tung Phan \& Chang Won Jung
}

An electromagnetic pulse (EMP) with high energy can damage electronic equipment instantly within a wide range of thousands of kilometers. Generally, a metal plate placed inside a thick concrete wall is used against an EMP, but it is not suitable for an EMP shielding window, which requires not only strong shielding effectiveness (SE) but also optical transparency (OT). In this paper, we propose a very thin and optically transparent structure with excellent SE for EMP shielding window application. The proposed structure consists of a saltwater layer held between two glass substrates and two metal mesh layers on the outside of the glass, with a total thickness of less than $1.5 \mathrm{~cm}$. The SE and OT of the structure are above $80 \mathrm{~dB}$ and 45\%, respectively, which not only meet the requirement of EMP shielding for military purposes but also retain the procedure of good observation. Moreover, the OT of the structure can be significantly improved using only one metal mesh film (MMF) layer, while the SE is still maintained high to satisfy the required SE for home applicants. With the major advantages of low cost, optical transparency, strong SE, and flexible performance, the proposed structure can be considered a good solution for transparent EMP shielding windows.

An electromagnetic pulse (EMP) is a short burst of electromagnetic (EM) wave generated by a nuclear explosion with very high energy that can damage electronic equipment instantly within a wide range. An EMP can destroy electronic equipment, communication systems, radio stations, and radar from a distance of thousands of kilometers from the center of the explosion ${ }^{1}$. The mechanism behind the damage caused by an EMP is that when an EMP arrives at a device, it creates a very strong induced current that enters electronic circuits and destroys them ${ }^{1,2}$. From the point of military defense, an EMP can neutralize and put at risks national and international infrastructure facilities, such as electrical grids and communication networks ${ }^{3}$. Moreover, it can cause severe damage to confidential military intelligence systems by paralyzing military communication equipment ${ }^{4}$. Therefore, developing EMP shielding methods to minimize damage is highly recommended.

Most previous research on the EMP shielding method focused on the SE of concrete structures and multiwaveguides with hexagonal/circular structures because of their excellent shielding performance ${ }^{5-7}$. Concrete structures are the basic frame of EMP shielding facilities to protect shielding rooms from being destroyed by external physical influence. In general, when the concrete structure is constructed, a shielding room is manufactured by metal plates placed on the inside of the concrete structure. However, the use of such a conventional material is not suitable for optical windows to utilize for aeronautic, medical, civilian, and research facilities, which require not only strong SE but also optical transparency (OT). Even if the circular waveguide is optically transparent, its large size leads to a thick structure (at least $15 \mathrm{~cm}$ ), therefore making it difficult to install on an optical window. Conversely, transparent electrodes (TEs), such as indium tin oxide (ITO) ${ }^{8}$, graphene ${ }^{9}$, carbon nanotubes $(\mathrm{CNTs})^{10}$, metallic nanowires $(\mathrm{MNWs})^{11}$, and metal mesh film $(\mathrm{MMF})^{8,12}$, have also been studied because of their favorable OT and thin-film structure. However, these TEs show a low shielding performance compared with the requirement of EMP applications. Therefore, the development of a transparent EMP shielding structure is imperative, but the existing great challenge is how to balance the inherent contradiction between the high OT and strong $\mathrm{SE}^{8}$. Specifically, the required EMI SE for EMP shielding is very high at $60 \mathrm{~dB}$ for general 


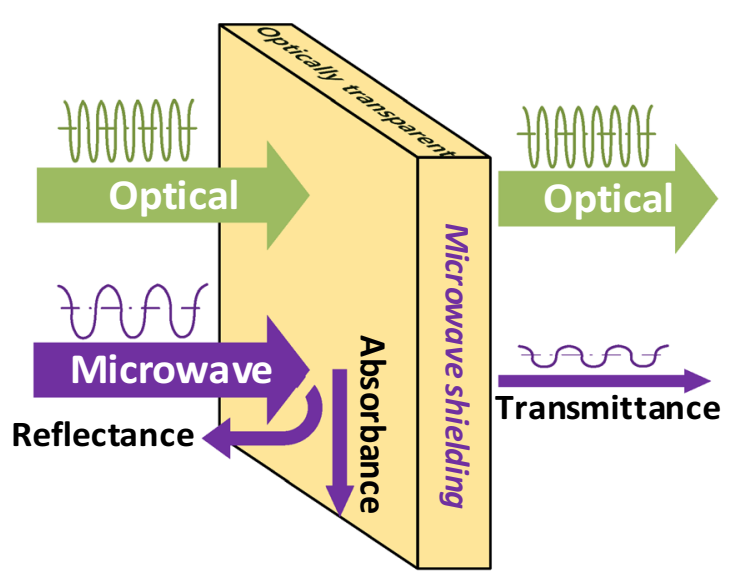

(a)

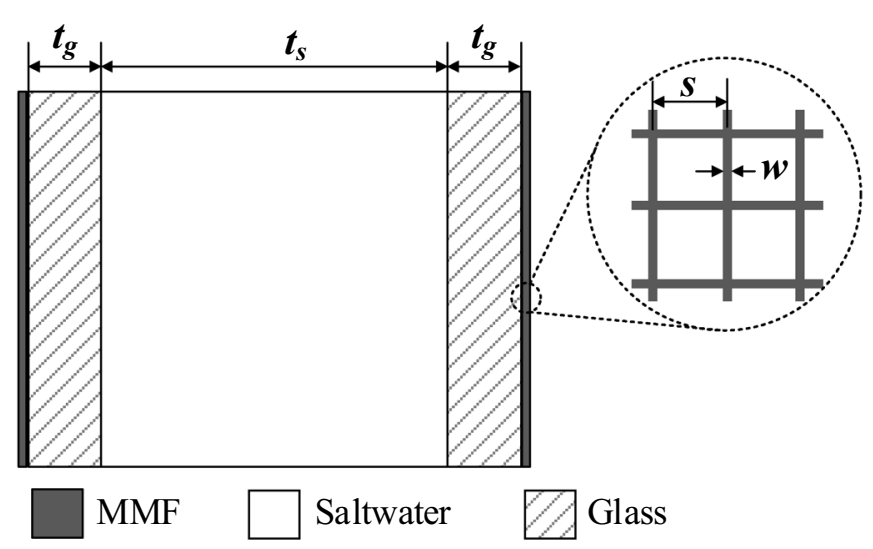

(b)

Figure 1. (a) Schematic diagram of the EMP shielding window; (b) geometry of the MMF/glass/saltwater/ glass/MMF structure.

purposes and $80 \mathrm{~dB}$ for military applications ${ }^{13,14}$. Therefore, the design of transparent and effective EMP shielding structures remains challenging.

This research proposes a transparent EMP shielding solution replacing conventional methods using a metal plate or a concrete wall. The proposed method consists of a high-salinity saltwater layer, which is held between two transparent quartz glass layers. To enhance the SE of the structures, one or two metal mesh layers are added to the left and right sides of the quartz glass layers. The proposed method has advantages over its conventional counterparts, including OT, low cost, and ease in fabrication. It is expected to reduce much of the construction costs for EMP-shielded rooms.

\section{Concept and schematic}

A schematic illustration of the EMP shielding window is shown in Fig. 1a. Most of the EM pulses in radio frequency are reflected and absorbed by the window and rarely pass through it, whereas visible light can transmit without any being affected. To do this task, the window must consist of components that are not only optically transparent but also microwave shielded.

Figure $1 \mathrm{~b}$ shows the geometry of the MMF/glass/saltwater/glass/MMF structure. As shown in Fig. 1b, the TE consists of a saltwater layer (thickness $t_{s}$ ) held between two clear glass quartz layers (thickness $t_{g}$ ). To enhance the SE of the structure to meet the requirements of EMP applications, we tape two MMF layers on the outer sides of each glass layer. The mesh width $(w)$ and mesh space $(s)$ of the MMF are $0.021 \mathrm{~mm}$ and $0.234 \mathrm{~mm}$, respectively. Owing to the good transparency of MMF, glass, and saltwater, we expect the OT of the multilayer structure to be retained at high.

\section{Optical analysis}

Salinity and thickness of saltwater layer are two key parameters that can affect on the OT of the proposed structure. Therefore, first of all we investigate the OT of a glass/saltwater/glass structure with different values of the salinity and thickness. Figure 2 a shows the measured OT of glass/saltwater/glass with different salinities and thicknesses of the saltwater layer to study the effect of these parameters on the OT of the structure. The thickness of the saltwater layer varies at 1,10 , and $20 \mathrm{~mm}$, and the salinity varies at 35,80 , and $200 \mathrm{ppt}$, corresponding to the conductivity of 5,10 , and $20 \mathrm{~S} / \mathrm{m}$, respectively. The OT of the structure decreases with the increase in the thickness of the saltwater layer. This can be explained using Beer-Lambert's law, in which attenuation of the light in saltwater at certain salinity is proportional to the thickness of the saltwater layer.

However, the OT of the structure is nearly unchanged with the increase in salinity. Specifically, when the thickness of the saltwater layer is $1 \mathrm{~mm}$ (the absorption can be ignored), the OT of the structure slightly increases. This can be explained by the fact that as salinity increases, the refractive index of saltwater also increases toward the refractive index of the glass (the refractive index of glass is 1.47$)^{15,16}$. Therefore, the refractive index matching between saltwater and glass improves with the increase in salinity, resulting in a decrease in the reflection of light at the interface between them and an increase in transmission through a multilayer structure ${ }^{17}$.

When the saltwater is thin (i.e., $1 \mathrm{~mm}$ ), the absorption is small and can be ignored. Therefore, the total OT slightly increases with the increase in salinity. However, when the saltwater layer becomes thicker (i.e., 10 and $20 \mathrm{~mm}$ ), the absorption is significant, which reduces the transmission ${ }^{18}$. This explains why the OT of the multilayer structure is nearly unchanged with the increase in salinity when the thickness is 10 and $20 \mathrm{~mm}$.

Figure $2 \mathrm{~b}$ shows the measured OT of different electrodes and structures in the visible range. First, we measure the OT of an MMF layer and a glass/saltwater/glass structure for reference. Second, one and two MMF layers are alternately taped to the glass/saltwater/glass structure, and the OT of these multilayer structures are measured. The thickness of the saltwater $\left(t_{s}\right)$ and quartz glass layers $\left(t_{g}\right)$ is fixed at $10 \mathrm{~mm}$ and $2 \mathrm{~mm}$, respectively. The salinity $(S)$ of saltwater is $200 \mathrm{ppt}$. 


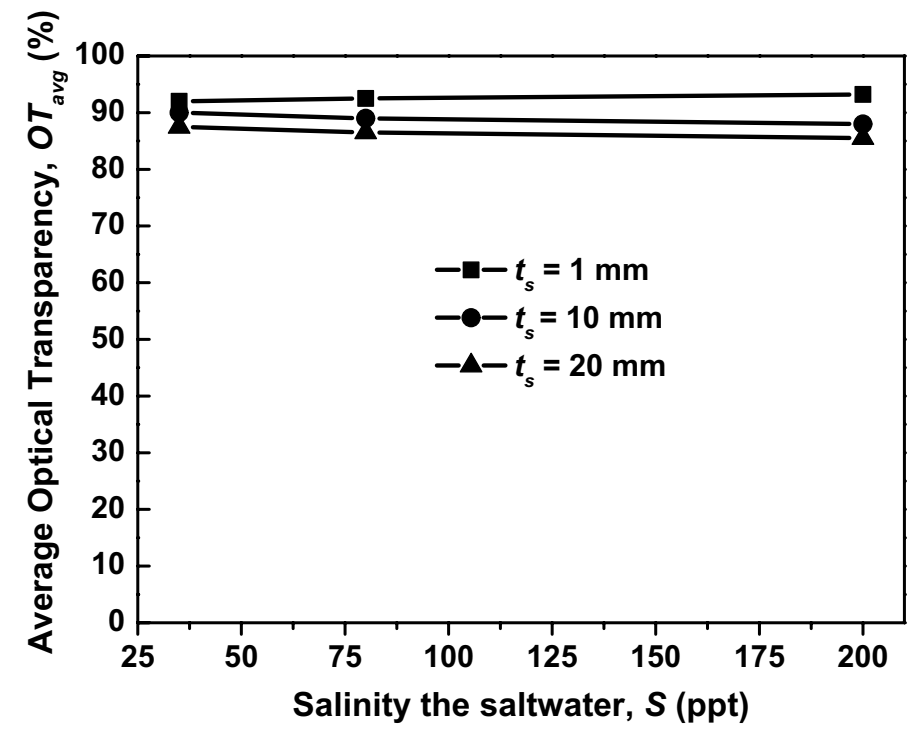

(a)

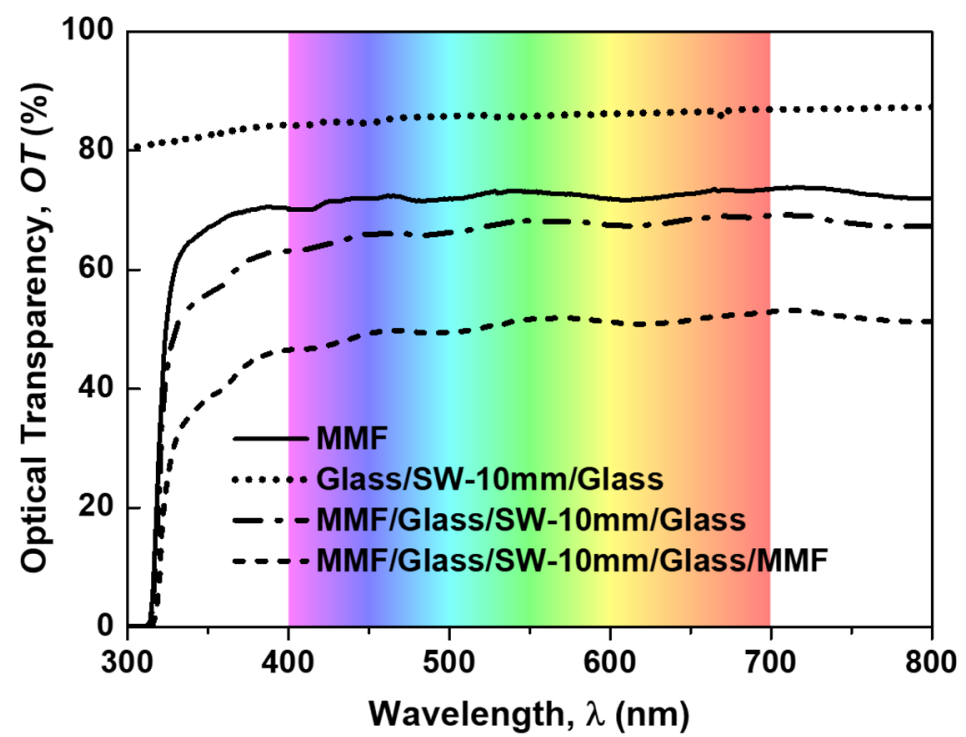

(b)

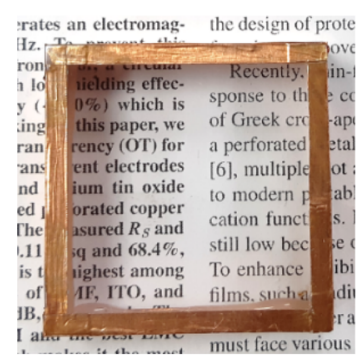

(c)

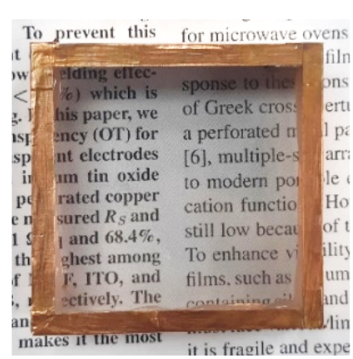

(d)

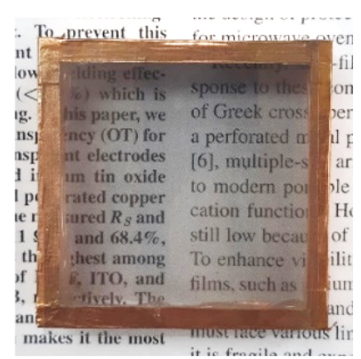

(e)

Figure 2. (a) Average OT of the glass/saltwater/glass structure with different thicknesses and salinities of the saltwater layer; (b) optical transparency spectra of transparent electrodes and structures in the visible range; real pictures of the TEs over texts $\left(t_{s}=10 \mathrm{~mm}\right)$ : (c) glass/saltwater/glass, (d) MMF/glass/saltwater/glass, and (e) MMF/glass/saltwater/glass/MMF. 
As shown in Fig. 2b, the OT of MMF is above $68 \%$, with an average value $\left(O T_{\text {avg }}\right)$ of $70 \%$ in the visible range, whereas the glass/saltwater/glass structure shows a very high OT of above $84 \%$, with an average value $\left(O T_{\text {avg }}\right)$ of $87.5 \%$ in the range, which is the highest among the TEs. Moreover, Fig. $2 \mathrm{~b}$ shows that by adding a single or double layer of MMF to the glass/saltwater/glass, the OT of the multilayer structures significantly decreases. The $O T_{\text {avg }}$ of the MMF/glass/saltwater/glass and MMF/glass/saltwater/glass/MMF structures in the visible range are $64 \%$ and $45 \%$, respectively.

Figures $2 \mathrm{c}$-e show real pictures of the three multilayer structures over text. The thickness of the saltwater in the three structures is $10 \mathrm{~mm}$, and the number of MMF layers varies at 0,1 , and 2 . The sample without MMF (Fig. 2c) shows a nearly perfect optical performance compared with the realistic one. As shown in Fig. 2d, e, even when the structures with one and two MMF layers show a darker color, they still retain a good enough optical performance for visual observation applications.

\section{EMI shielding of a multilayered saltwater}

Numerical simulation. In general, the EMI shielding mechanism of a material can be briefly described as the incident EM waves being either reflected or transmitted through the shielding material, but a considerable amount of EM waves may be attenuated because of absorption inside the material. The relevant shielding effectiveness parts for the reflection $\left(S E_{R}\right)$, absorption $\left(S E_{A}\right)$, and total $\left(S E_{T}\right)$ can be expressed as Eq. $(1)^{19}$

$$
S E_{T}=S E_{R}+S E_{A}
$$

where $S E_{R}$ and $S E_{A}$ are determined as Eqs. (2) and (3) ${ }^{19}$ :

$$
\begin{gathered}
S E_{R}=39.5+10 \log \left(\frac{\sigma}{2 \pi \mathrm{f} \mu}\right), \\
S E_{A}=8.7 t \sqrt{\pi f \mu \sigma},
\end{gathered}
$$

where $\sigma, \mu$, and $t$ are the electrical conductivity, magnetic permeability, and thickness of the shielding material, respectively. $f$ is the frequency of the incident EM wave.

To examine the EM shielding mechanism of the saltwater layer, we extract the magnitude distribution of the E-field of the incident EM wave when it propagates along with the thickness of the saltwater layer. As shown in Fig. 3a, the incident EM wave with a frequency of $8 \mathrm{GHz}$ (@wavelength $\lambda_{0}$ of $37.5 \mathrm{~mm}$ ) propagates along the $\mathrm{z}$-axis from the leftmost to the rightmost of the saltwater layer. Note that when the EM wave propagates in saltwater with a permittivity of 81 , the wavelength of the EM wave is $\lambda_{s}=4.17 \mathrm{~mm}$. The thickness of the saltwater layer is set to $2.1 \mathrm{~mm}\left(\lambda_{s} / 2\right)$. Therefore, the E-field distribution is extracted at phases $0^{\circ}, 90^{\circ}$, and $180^{\circ}$, corresponding to the magnitude at the leftmost, center, and rightmost points $\left(E_{0}, E_{90}, E_{180}\right)$, respectively. The magnitude of the incident EM wave significantly decreases when it propagates from the leftmost to the rightmost of the saltwater layer $\left(E_{0}>E_{90}>E_{180}\right)$. This observation confirms that the absorption contributes crucially to the total EM shielding of the saltwater layer. The EM shielding mechanism of the saltwater layer is different from the metal mesh, in which the reflection mainly contributes to the total EM shielding ${ }^{8}$. A similar phenomenon of EM absorption was reported in the literature ${ }^{20}$.

The shielding effectiveness (SE) of saltwater is a function of its conductivity, which is determined from salinity. Figure $3 \mathrm{~b}$ shows the simulated SE of the glass/saltwater/glass $\left(t_{s}=10 \mathrm{~mm}\right)$ with different salinities. The salinities of saltwater are 35,80 , and $200 \mathrm{ppt}$, which correspond to the conductivity of 5,10 , and $20 \mathrm{~S} / \mathrm{m}$ at a room temperature of $25^{\circ} \mathrm{C}$. The SE of the glass/saltwater/glass structure is significantly improved when the salinity increases from 35 to 200 ppt. The increase in the SE of saltwater with the increase in salinity can be explained, as shown in Eqs. (2)-(3), because both reflection and absorption increase with the increase in conductivity of saltwater. Note that the saturated salinity of saltwater at room temperature is $263 \mathrm{ppt}^{21}$. Therefore, we maintain the salinity of the saltwater layer as high as $200 \mathrm{ppt}$ to ensure that the solution cannot be saturated, while the SE is retained at high.

The thickness of the saltwater layer is a basic parameter determining the shielding performance of the glass/ saltwater/glass structure. When the thickness of the saltwater layer is less than the skin depth, the shielding performance decreases. In this work, we investigate the effect of the thickness of the saltwater layer on the shielding performance of the glass/saltwater/glass structure, as shown in Fig. 3c. The SE of the glass/saltwater/ glass structure is determined using different thicknesses of the saltwater layer while the salinity is fixed at 200 ppt. The SE of the multilayer structure significantly increases with the increase in the thickness of the saltwater layer from 1 to $20 \mathrm{~mm}$. This can be explained by the sheet resistance of the saltwater layer decreasing with the increase in thickness, which contributes to the improvement of the SE. Even the SE can be significantly improved by increasing the thickness of the saltwater layer, as a too-thick structure can limit its application to the optical window. Moreover, the SE of a $20-\mathrm{mm}$ saltwater multilayer is below $60 \mathrm{~dB}$, which is not strong enough for most EMP shielding applications. Therefore, we propose using a 10-mm-thick saltwater layer with MMF to overcome this problem.

Figure 3d shows the SE of glass/saltwater/glass structure with one and two MMF layers. The SE of the MMF and glass/saltwater/glass structure without MMF is also determined for comparison. The saltwater layer shows a good SE above $40 \mathrm{~dB}$ in the whole C-band, and it is much better than the MMF, which has an average SE of $19 \mathrm{~dB}$ in the band. Although the saltwater layer shows a high SE, it is not strong enough for EMP applications, which require an SE of at least $60 \mathrm{~dB}$. Therefore, we alternately install a single and double layer of MMF on the saltwater layer to improve the SE value. As expected, the SE of the TEs is significantly improved, as shown in Fig. 3d. The MMF/glass/saltwater/glass and MMF/glass/saltwater/glass/MMF show SE values of above $60 \mathrm{~dB}$ 

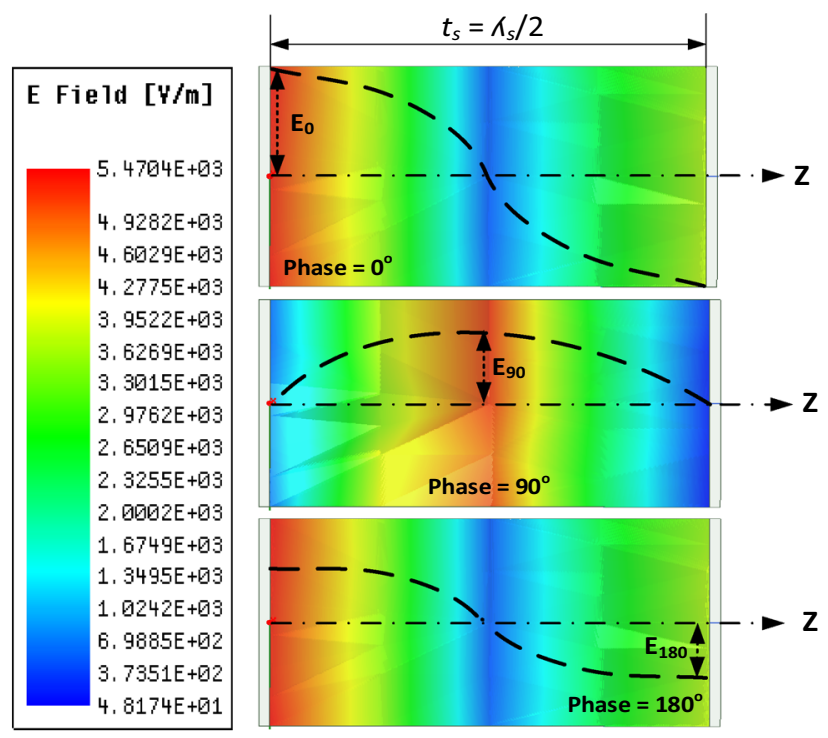

(a)

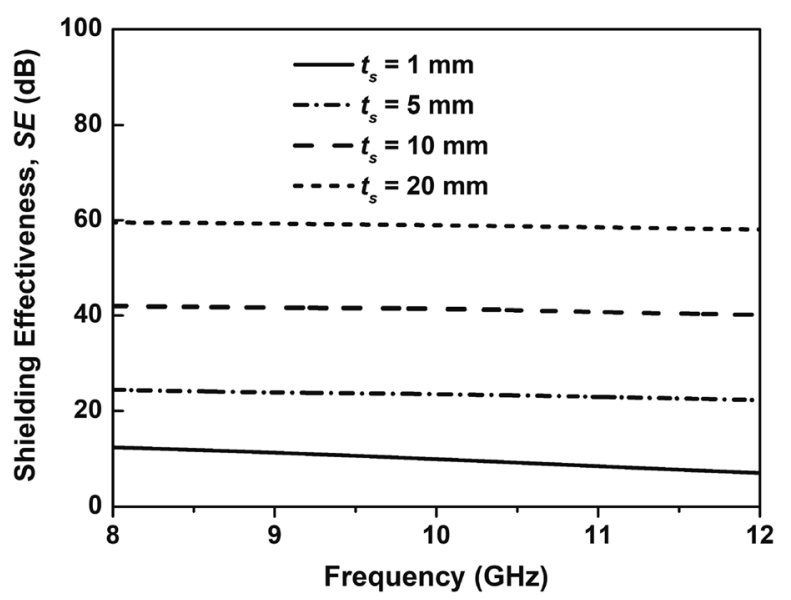

(c)

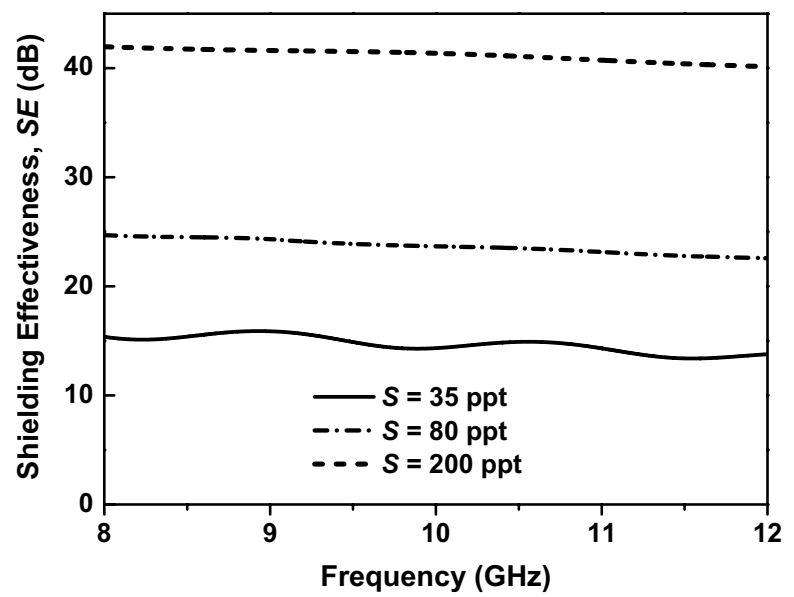

(b)

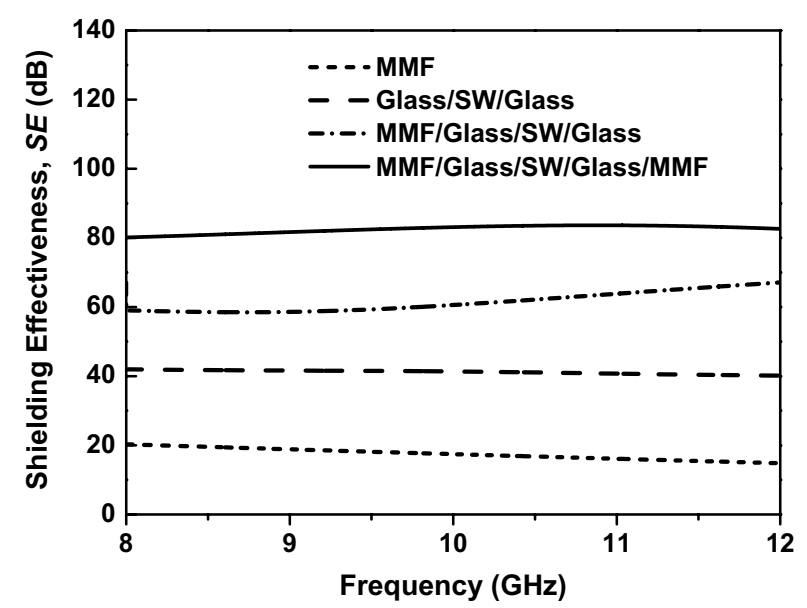

(d)

Figure 3. (a) E-field distribution of the incident EM wave along with the thickness of the saltwater layer with a thickness of a half wavelength of the EM wave in saltwater $\left(\lambda_{s}\right)$; SE of the planar saltwater in the C-band: (b) at different salinities $\left(t_{s}=10 \mathrm{~mm}\right)$, (c) at different thicknesses $(\mathrm{S}=200 \mathrm{ppt})$; (d) SE of the four types of TEs in the C-band.

and $80 \mathrm{~dB}$ in the entire interest band, respectively. These values are suitable for EMP applications for commercial and military purposes, respectively.

Experiment test. To verify the simulation results, the measurement of the structure under test (SUT) is conducted using a pair of waveguide-to-coaxial adapters, as shown in Fig. 4a. The SUTs are MMF/glass/saltwater/glass and MMF/glass/saltwater/glass/MMF, with a thickness and salinity of saltwater of $10 \mathrm{~mm}$ and $200 \mathrm{ppt}$, respectively. The saltwater layer is held between two clear quartz glass layers $\left(t_{g}=2 \mathrm{~mm}, \varepsilon=4.3, \tan \delta=0\right)$. The single and double layers of MMF are alternately installed on the glass layers to fabricate the SUTs, as depicted in Fig. 1b. The MMF layers are very thin $(0.005 \mathrm{~mm})$ and can be ignored. Therefore, the total thickness of the multilayered structure is $14 \mathrm{~mm}$. To avoid EM wave leakages between two adapters, we use copper tape to block this gap. Note that the SE can be determined from the power transmission coefficient $\left(S_{21}\right)$ in $\mathrm{dB}$ as $S E=\left|S_{21}\right|$, which is determined using a vector network analyzer connected to the adapters, as shown in Fig. $4 \mathrm{a}$.

Figure $4 \mathrm{~b}$ shows the measured SE of the TEs, which is in good agreement with the simulation. The measured SE of the structures slightly increases with the increase in frequency. This can be explained by the incident EM wave over $100 \mathrm{kHz}$ : attenuation in saltwater increases with an increase in frequency, leading to a decrease in the wave transmitted through the structure ${ }^{22,23}$. Moreover, the SE of the saltwater layer also slightly increases with an increase in frequency when the thickness of the glass layers $\left(t_{g}\right)$ is smaller than a quarter wavelength of the EM wave in the C-band ${ }^{24}$. 


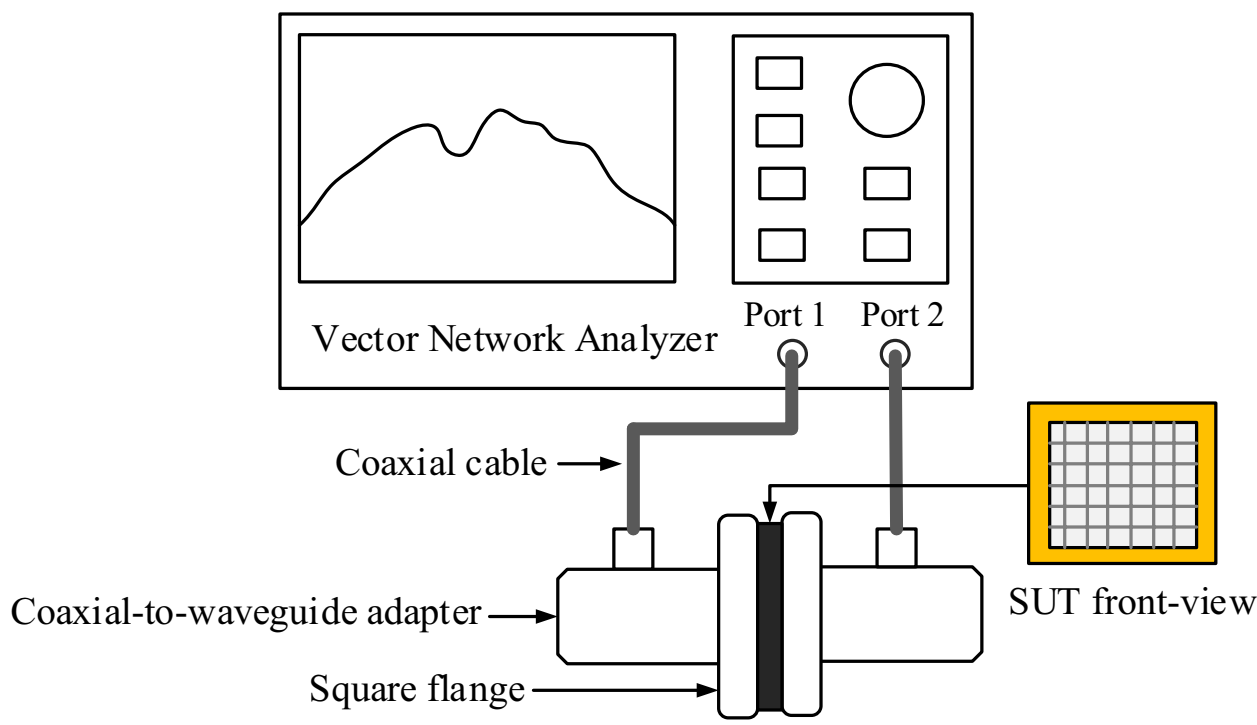

(a)

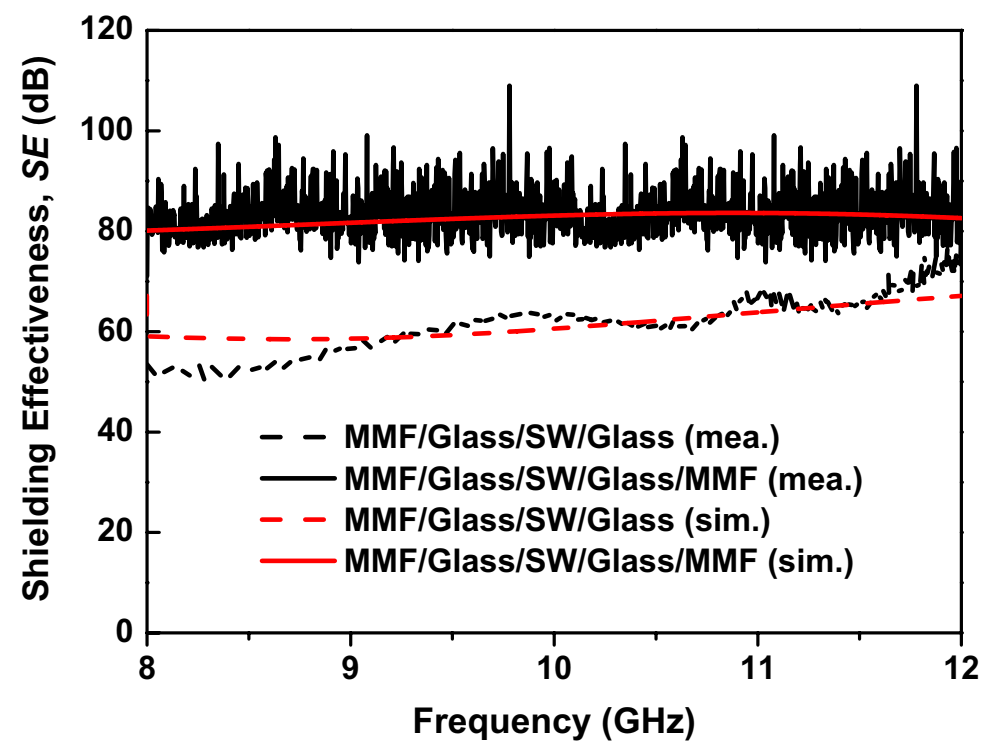

(b)

Figure 4. (a) Setup for the SE measurement of the structures; (b) simulated and measured SE of the saltwater TEs with single and double MMF $\left(t_{s}=10 \mathrm{~mm}\right)$.

\begin{tabular}{|l|l|l|l|}
\hline TEs & Thickness $(\mathbf{m m})$ & $\boldsymbol{O T}_{\text {avg }}(\%)$ & $\boldsymbol{S E}_{\text {avg }}(\mathbf{d B})$ \\
\hline MMF & 0.005 & 70 & 19 \\
\hline Glass/saltwater/glass & 14 & 85 & 41 \\
\hline MMF/glass/saltwater/glass & 14.005 & 64 & 61 \\
\hline MMF/glass/saltwater/glass/MMF & 14.01 & 45 & 82 \\
\hline
\end{tabular}

Table 1. Summarized OT and SE of the proposed structures.

The saltwater layer with a double layer of MMF shows a very strong SE over $80 \mathrm{~dB}$, with an average value $\left(S E_{\text {avg }}\right)$ of $82 \mathrm{~dB}$ in the C-band from 8 to $12 \mathrm{GHz}$. Although the measured $\mathrm{SE}$ of the saltwater layer with a single layer of MMF is significantly lower than that of the double-layer MMF, the SE is over $60 \mathrm{~dB}$ with $S E_{\text {avg }}$ of $61 \mathrm{~dB}$ in the band, which is suitable for EMP shielding for commercial purposes. Table 1 summarizes the average OT and SE of the TEs. 


\begin{tabular}{|l|l|l|l|l|l|}
\hline References & Transparent electrode/structure & Thickness $(\mathbf{m m})$ & Frequency $(\mathbf{G H z})$ & OT $(\%)$ & SE $(\mathbf{d B})$ \\
\hline 7 & Circular multi-waveguide & 250 & 1 & N/A & $>80$ \\
\hline 24 & Double-layer metal mesh & 6.0012 & $0.15-5$ & 76.35 & 37.61 \\
\hline 25 & Silver nanowires & N/A & $8-12$ & 91.3 & 28 \\
\hline 26 & Crackle template-based metallic mesh & 7 & $12-18$ & 91 & 26 \\
\hline 27 & Graphene/metal mesh & N/A & $12-18$ & 90 & 14.1 \\
\hline \multirow{2}{*}{ This work } & MMF/glass/saltwater/glass & 14.005 & \multirow{2}{*}{$8-12$} & 64 & 61 \\
\cline { 2 - 6 } & MMF/glass/saltwater/glass/MMF & 14.01 & 45 & 82 \\
\hline
\end{tabular}

Table 2. Comparison of the proposed structures with other studies.

Table 2 shows the comparison between the proposed structures with the existing transparent electrodes in terms of thickness, working frequency, optical, and SE performance. Most existing TEs are nanowire-based and graphene-based structures that show a very high OT (>90\%). However, the SE of these TEs is low $(<30 \mathrm{~dB})$, which is not suitable for EMP applications. The double metal mesh layer shows a higher SE of $37.61 \mathrm{~dB}$, but it is still not strong enough for EMP applications. On the other hand, the circular multi-waveguide has excellent shielding performance of above $80 \mathrm{~dB}$, but it has a large size, which is a major disadvantage for applications. Compared with the existing transparent TEs and structures, our proposed structure, which is very thin, shows excellent SE that meets the requirement of EMP shielding windows for commercial and military purposes, while the OT performance is retained for the good observation requirement, thus making it the most suitable candidate for EMP shielding window applications.

\section{Conclusions}

We have successfully fabricated a very thin transparent multilayered structure (less than $1.5 \mathrm{~cm}$ ) demonstrating excellent EMI shielding performance and sufficient optical transmittance. The major reason for developing a thin and transparent structure is that conventional shielding, such as concrete with a metal plate, is too thick and nontransparent, thus making it inappropriate for applications such as optical windows. The proposed structure consists of a saltwater layer held between two clear glass layers. One or two metal mesh film layers are taped on the outer side of the glasses to enhance the shielding performance of the structure. The OT and SE of the proposed structure are carefully examined by simulation and an experiment, and good agreement is achieved. Specifically, the multilayer structures exhibit a uniform OT of above $44 \%$ in the visible band and a strong SE of over $80 \mathrm{~dB}$ in the C-band. Moreover, the OT of the structure can be significantly improved by using only one MMF layer, with the SE maintained high to meet the requirement of the EMP shielding for commercial purposes. The SE can be strengthened by increasing the thickness or salinity of the saltwater with a negligible decrease in the OT. With the major advantages of low cost, optical transparency, strong SE, and flexible performance, the multilayered structure using saltwater in glass and MMF on glass can be considered a good solution for transparent EMP shielding applications.

\section{Methods}

Materials. The ionized salt used in this study was kindly provided by the manufacturer. We prepared the saltwater solution by dissolving the salt in pure water at a room temperature of $25^{\circ} \mathrm{C}$ following two steps: (1) pour $100 \mathrm{ml}$ water, which corresponds to $100 \mathrm{~g}$, into a $200 \mathrm{ml}$ glass beaker, and (2) add salt to the beaker and stir. The amount of salt was controlled to achieve the desired salinity using the relationship between them as follows: $m_{s}=100 S /(1000-S)$, where $m_{s}$ is the amount of salt in grams, and $S$ is the salinity of the saltwater solution in parts per thousand (ppt).

Simulation. The simulation of the EMI SE of the TEs was conducted using Ansys HFSS (high frequency structure simulator) R2019.1. As shown in Fig. 5, the TEs were placed between two wave ports to determine the power transmission $\left(S_{21}\right)$ from port 1 to port 2. The simulated SE of the TEs was determined from the $S_{21}($ in $\mathrm{dB})$ as $S E=\left|S_{21}\right|$.

Measurement. The OT measurement was conducted using an ultraviolet-visible spectrophotometer (T60 model, PG Instruments Limited Co., UK) connected to a computer. The measurement SE of the TEs in the C-band was measured using a pair of waveguide-to-coaxial adapters (90WCAS) connected to ports 1 and 2 of a vector network analyzer (model E8364C, Keysight Technologies Co., US), as shown in Fig. 4a. The opening cross-section of the adapters was $22.5 \mathrm{~mm} \times 10 \mathrm{~mm}$, corresponding to a bandwidth of 8-12 GHz. The SUT had the same dimension fit to the outer size (cover flange) of the adapters $(45 \mathrm{~mm} \times 45 \mathrm{~mm}$ ). The SUT was fabricated using 2 -mm-thick quartz glass $(\varepsilon=4.3, \tan \delta=0)$ as the substrate and a 10 -mm-thick saltwater layer $(S=200 p p t @ \sigma=20 \mathrm{~S} / \mathrm{m})$ as the conductive layer. The total thickness of the SUT was $14 \mathrm{~mm}$. Therefore, to avoid leakage waves, copper tape was used to block this gap. The measurement procedure of the SUT includes two steps: (1) connect the two adapters directly and measure the transmission coefficient $S_{210}$, and (2) separate the two adapters using the SUT and measure transmission coefficient $S_{21 S}$. The measured SE of the SUT was determined from the measured transmission coefficients as $S E=\left|S_{21 S}\right|-\left|S_{210}\right|$. 


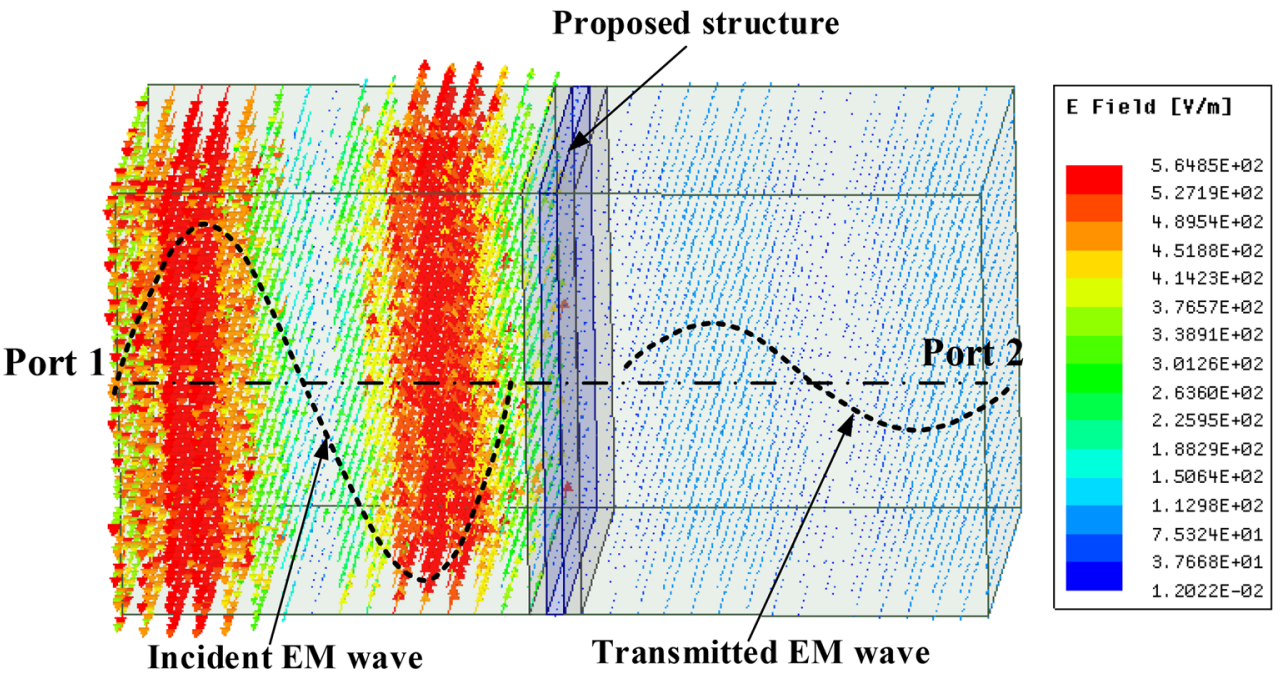

Figure 5. Simulation setup for the SE of proposed structures using Ansys HFSS R2019.1.

Received: 22 October 2020; Accepted: 28 December 2020

Published online: 28 January 2021

\section{References}

1. Kim, H. Truth of EMP threat and development plan. DefenseTech 414, 98-103 (2013).

2. Choi, T. \& Cho, W. Countermeasure of electromagnetic pulse (EMP). DenfenseTech 157, 54-59 (1992).

3. Jacob, G. The Swiss EMP concept of general defense. IEEE Antennas Propag. Soc. Newsl. 29, 5-10 (1987).

4. Fugetsu, B. et al. Electrical conductivity and electromagnetic interference shielding efficiency of carbon nanotube/cellulose composite paper. Carbon 46, 1256-1258 (2008).

5. Fei, G., Bihua, Z., Cheng, G., \& Hailin, C.: Analysis of shielding effectiveness of conductive cement-based materials in HEMP environment. In Proceeding of the 2008 International Conference on Microwave and Millimeter Wave Technology (ICMMT 2008), Nanjing, China, 21-24 April 2008.

6. Scott, W. P. \& Daniel, N. A. Analysis, modeling, and measurement of shielding effectiveness for a cylindrical waveguide with a hexagonal insert structure. ACES J. 33, 8 (2018).

7. Yoon, S., Jeong, K., Mumtaz, S. \& Choi, E. H. Electromagnetic pulse shielding effectiveness of circular multi-waveguides for fluids. Results Phys. 16, 102946 (2020).

8. Tung, P. D. \& Jung, C. W. High optical visibility and shielding effectiveness metal mesh film for microwave oven application. IEEE Trans. Electromagn. Compat. 62(4), 1706-1781 (2020).

9. Gupta, T. K. et al. Multi-walled carbon nanotube graphene-polyaniline multiphase nanocomposite with superior electromagnetic shielding effectiveness. Nanoscale 6, 842-851 (2014).

10. Al-Saleh, M. H. et al. EMI shielding effectiveness of carbon based nanostructured polymeric materials: A comparative study. Carbon 60, 146-156 (2013).

11. Preston, C. et al. Silver nanowire transparent conducting paper-based electrode with high optical haze. J. Mater. Chem. C 2, 1248-1254 (2014).

12. Xu, et al. Cross-wavelength invisibility integrated with various invisibility tactics. Sci. Adv. 6, eabb3755 (2020).

13. MIL-STD-188/125. High-altitude electromagnetic pulse (HEMP) protection for ground-based C4I facilities performing critical, time-urgent missions part 1-fixed facilities. 2005.

14. ITU-T K.48. EMC requirements for telecommunication equipment-product family recommendation. 2006.

15. Quan, X. \& Fry, E. S. Empirical equation for the index of refraction of seawater. Appl. Phys. 34, 3477-3480 (1995).

16. Ghosh, G. Dispersion-equation coefficients for the refractive index and birefringence of calcite and quartz crystals. Opt. Commun. 163, 95-102 (1999).

17. Franklin, J. \& Wang, Z. Y. Refractive index matching: A general method for enhancing the optical clarity of a hydrogel matrix. Chem. Mater. 14, 4487-4489 (2002).

18. Jerlov, N. G. Marine Optics 2nd edn, 14 (Elsevier, New York, 1976).

19. Al-Saled, M. H. \& Sundararaj, U. Electromagnetic interference shielding mechanisms of CNT/polymer composites. Carbon 47, 1738-1746 (2009).

20. Pang, et al. Water-based metamaterial absorbers for optical transparency and broadband microwave absorption. J. Appl. Phys. 123, 155106 (2018)

21. Kalashnikov, P. A., Shkvorets, I. Y. \& Egorov, Y. A. Metrological support of salinity, temperature and pressure measurements, using CTD-systems. Phys. Oceanogr. 9, 71-77 (1998).

22. Lee, S., Yoon, H., Baik, K. \& Jan, B. Emulator for generating heterogeneous interference signals in the Korean RFID/USN frequency band. J. Electromagn. Eng. Sci. 18(4), 254-260 (2018).

23. E. Jimenez, G. Quintana, P. Mena, P. Dorta, I. P. Alvarez, S. Zazo, M., \& Perez, E. Quevedo. Investigation on Radio Wave Propagation in Shallow Seawater: Simulations and Measurements. In 2016 IEEE Third Underwater Communications and Networking Conference (UComms), Lerici, 1-5 (2016).

24. Zhang, Y. et al. Double-layer metal mesh etched by femtosecond laser for high-performance electromagnetic interference shielding window. RSC Adv. 9, 22282 (2019).

25. Zhu, X. et al. Higly efficient and stable transparent electromagnetic interference shielding films based on silver nanowires. Nanoscale 12, $14589(2020)$.

26. Han, Y. et al. Crackle template based metallic mesh with highly homogeneous light transmission for high-performance transparent EMI shielding. Sci. Rep. 6, 25601 (2016). 
27. Han, Y., Liu, Y., Han, L., Lin, J. \& Jin, P. High-performance hierarchical graphene/metal-mesh film for optically transparent electromagnetic interference shielding. Carbon 115, 34-42 (2017).

\section{Acknowledgements}

This work was supported by the National Research Foundation (NRF) of Korea Grant funded by the Korean Government (no. 2016R1D1A1B02012957).

\section{Author contributions}

D.T.P. and C.W.J. described the methodology; D.T.P. conducted the simulations and experiments and analyzed the results; D.T.P. wrote the original draft; C.W.J. reviewed and edited the draft. C.W.J. provided supervision. All authors reviewed the manuscript.

\section{Competing interests}

The authors declare no competing interests.

\section{Additional information}

Correspondence and requests for materials should be addressed to C.W.J.

Reprints and permissions information is available at www.nature.com/reprints.

Publisher's note Springer Nature remains neutral with regard to jurisdictional claims in published maps and institutional affiliations.

(c) (1) Open Access This article is licensed under a Creative Commons Attribution 4.0 International License, which permits use, sharing, adaptation, distribution and reproduction in any medium or format, as long as you give appropriate credit to the original author(s) and the source, provide a link to the Creative Commons licence, and indicate if changes were made. The images or other third party material in this article are included in the article's Creative Commons licence, unless indicated otherwise in a credit line to the material. If material is not included in the article's Creative Commons licence and your intended use is not permitted by statutory regulation or exceeds the permitted use, you will need to obtain permission directly from the copyright holder. To view a copy of this licence, visit http://creativecommons.org/licenses/by/4.0/.

(C) The Author(s) 2021 